\title{
Antibiotic Resistance and Molecular Epidemiological Characteristics of Streptococcus agalactiae Isolated from Pregnant Women in Guangzhou, South China
}

\author{
Zhaomin Cheng, ${ }^{1,2}$ Pinghua Qu, ${ }^{1,2}$ Peifeng Ke, ${ }^{1,2}$ Xiaohan Yang, ${ }^{3}$ Qiang Zhou, ${ }^{1,2}$ Kai Lan, ${ }^{1,2}$ \\ Min He, ${ }^{1,2}$ Nannan Cao, ${ }^{1,2}$ Sheng Qin, ${ }^{1,2}$ and Xianzhang Huang $\mathbb{D}^{1,2}$ \\ ${ }^{1}$ The Second Clinical College, Guangzhou University of Chinese Medicine, Guangzhou, China \\ ${ }^{2}$ Department of Laboratory Medicine, Guangdong Provincial Hospital of Chinese Medicine, Guangzhou, China \\ ${ }^{3}$ Department of Prenatal Diagnosis, Guangdong Provincial Maternity and Children's Hospital, Guangzhou, China
}

Correspondence should be addressed to Xianzhang Huang; huangxz020@163.com

Received 27 September 2019; Revised 16 January 2020; Accepted 7 March 2020; Published 30 April 2020

Academic Editor: Bruno Pozzetto

Copyright (c) 2020 Zhaomin Cheng et al. This is an open access article distributed under the Creative Commons Attribution License, which permits unrestricted use, distribution, and reproduction in any medium, provided the original work is properly cited.

\begin{abstract}
Streptococcus agalactiae colonization in pregnant women can cause postpartum intrauterine infections and life-threatening neonatal infections. To formulate strategies for the prevention and treatment of S. agalactiae infections, we performed a comprehensive analysis of antibiotic resistance and a molecular-based epidemiological investigation of S. agalactiae in this study. Seventy-two S. agalactiae strains, collected from pregnant women, were subjected to antibiotic susceptibility tests; then, the screened erythromycin and clindamycin nonsusceptible isolates were used for macrolides and clindamycin resistance genes detection, respectively. Detection of resistance genes, serotyping, and determination of virulence genes were performed by polymerase chain reaction. The clonal relationships among the colonized strains were evaluated by multilocus sequence typing. Matrix-assisted laser desorption/ionization time-of-flight mass spectrometry (MALDI-TOF MS) mass peak analysis was performed to discriminate the specific sequence types (STs). In our study, $69.4 \%$ and $47.2 \%$ of the strains were nonsusceptible to erythromycin and clindamycin, respectively; the multidrug resistance rate was $66.7 \%$. All erythromycin nonsusceptible strains harbored resistance genes, whereas only $52.9 \%$ of the clindamycin nonsusceptible strains possessed the linB gene. Erythromycin resistance was mainly mediated by the ermB or $m e f A / E$ genes. Four serotypes were identified, and the most common serotype was serotype III (52.8\%), followed by Ib (22.2\%), Ia (18.0\%), and II (4.2\%). All the strains were divided into 18 STs that were assigned to nine clonal complexes. Most of the major STs were distributed into specific serotypes, including ST19/serotype III, ST17/serotype III, ST485/serotype Ia, ST862/serotype III, and ST651/serotype III. Analysis of virulence genes yielded seven clusters, of which $b c a-c f b-s c p B-\operatorname{lmb}(61.6 \%)$ was the predominant virulence gene cluster. Among all ST strains distributed in this region, only the ST17 strains had a mass peak at $7620 \mathrm{Da}$. The outcomes of this study are beneficial for the epidemiological comparison of colonized $S$. agalactiae in different regions and may be helpful for developing the strategies for the prevention of $S$. agalactiae infection in Guangzhou. Furthermore, our results show that MALDI-TOF MS can be used for the rapid identification of the ST17 strains.
\end{abstract}

\section{Introduction}

Streptococcus agalactiae is a microorganism that commonly colonizes in the gastrointestinal and genitourinary tracts of healthy women $[1,2]$. S. agalactiae colonization in pregnant women is the main reason for intrauterine infection or transmission during parturition [3], which can cause serious neonatal infectious diseases, such as pneumonia, sepsis, and meningitis $[4,5]$. Many countries have already issued guidelines for the prevention of maternal and neonatal $S$. agalactiae infections [6-8]. These guidelines generally recommend that pregnant women with $S$. agalactiae 
colonization should receive intrapartum antibiotic prophylaxis (IAP) to reduce the incidence of these infections $[9,10]$. Currently, there are no official guidelines for $S$. agalactiae screening and prevention in China $[5,11]$. However, in recent years, S. agalactiae has become an important pathogen in Chinese obstetrics and neonatology [12]. In mainland China, the S. agalactiae colonization rate of pregnant women ranged from 3.7 to $14.5 \%$, and the incidence of invasive neonatal S. agalactiae diseases was 0.55-1.79 per 1000 live births, with a case fatality risk ranging from 6.5 to $7.1 \%$ [13]. The first-line antibiotic for IAP is penicillin $[9,10]$; however, since some people are allergic to it, macrolides (e.g., erythromycin) and lincosamides (e.g., clindamycin) are used as the alternative antibiotics [14]. Unfortunately, the resistance of S. agalactiae to macrolides and lincosamides is increasing worldwide $[4,9,11,15,16]$. Previous studies have shown that the ermB, ermTR, and $m e f A / E$ genes are involved in the resistance to macrolides [17], and the $\operatorname{lin} B$ gene has been linked to the resistance to lincosamides and moderate sensitivity to macrolides [18].

Capsular polysaccharide (CPS) is an important virulence factor of $S$. agalactiae, resulting in invasive infections $[16,19]$. According to the different antigenicity of the CPS $[20,21]$ or by the multiplex polymerase chain reaction (PCR) assay $[9,22], S$. agalactiae can be divided into 10 serotypes (Ia, Ib, and II to IX). Five of them (Ia, Ib, II, III, and V) are the predominant types of $S$. agalactiae that cause invasive infections $[16,23]$. The understanding of the serotype distribution is pivotal in developing CPS-based polyvalent vaccines $[16,21]$. In recent years, there have been a few reports on the serotype distribution of colonized $S$. agalactiae in China [11, 16, 24, 25]. Although there are some regional differences in the serotype distribution of $S$. agalactiae in these studies, similar common serotypes (III, Ia, V, and $\mathrm{Ib}$ ) were observed. Several studies have shown that the virulence of $S$. agalactiae depends on not only the CPS, but also the surface-exposed bacterial proteins [26-29]. Several molecular typing methods have been applied to epidemiological studies, such as pulsed-field gel electrophoresis (PFGE), random amplification of polymorphic DNA, and multilocus sequence typing (MLST), of which MLST is a robust and convenient method that results in exchangeable data in different labs [30]. The combination of MLST and serotyping is a valuable method for $S$. agalactiae typing. For instance, the ST17 strain, belonging to serotype III, was found to be "hypervirulent" S. agalactiae, apparently associated with neonatal invasive diseases [31,32]. Recently, whole-genome sequencing (WGS) has been used in genomic research of $S$. agalactiae $[9,33]$, such as accurate serotyping [34], in silico virulence investigation and antimicrobial susceptibility testing $[33,35]$, and speciation and evolutionary analysis [36]. However, at present, due to the high cost of WGS, it is not suitable for large sample analysis [34].

In recent years, matrix-assisted laser desorption/ionization time-of-flight mass spectrometry (MALDI-TOF MS), described as a "revolution in clinical microbiology" technology [32], has been widely used for bacterial identification based on protein fingerprints $[37,38]$, due to its accuracy, speed, and high throughput. This technology can also find characteristic proteomic biomarkers in some phylogenetic lineages of species and subspecies $[38,39]$. For example, it was reported that "hypervirulent" S. agalactiae ST17 and emerging ST-1 clones could be rapid detected by MALDITOF MS [39].

The purpose of this study was to investigate the antibiotic resistance and resistance genes, serotype distribution, virulence, and genotyping of $S$. agalactiae, isolated from pregnant women in Guangzhou and establish rapid screening methods for some STs, which may be beneficial for the prevention and control of $S$. agalactiae infection.

\section{Materials and Methods}

2.1. Strains Collection and Identification. 72 nonrepeating $S$. agalactiae strains were collected from pregnant women (35-37 weeks of pregnancy) in Guangdong Provincial Maternity and Children's Hospital, which is one of the major women's medical centers in Guangzhou, between January 2017 and December 2017. 1013 pregnant women were screened for colonized S. agalactiae during that time, and the carriage rate was $7.1 \%(72 / 1013)$.

The vaginal-rectal swabs collected from pregnant women were inoculated in Todd-Hewitt broth (bioMérieux, France), and the broth was incubated at $37^{\circ} \mathrm{C}$ in a $5 \% \mathrm{CO}_{2}$ environment for $24 \mathrm{~h}$. Afterwards, the samples were subcultured on Columbia agar, containing 5\% sheep blood (bioMérieux, France), and the agar was incubated in the same conditions for $18-24 \mathrm{~h}$. The suspected isolates were initially identified according to the following points: $\beta$-hemolysis (or nonhemolysis), colony morphology, and CAMP test [6]; the presumed $S$. agalactiae isolates were then confirmed by MALDI-TOF MS (VITEK ${ }^{\circledR}$ MS, bioMérieux, France).

2.2. DNA Extraction. The pure S. agalactiae cultures were harvested on Columbia agar, containing 5\% sheep blood. The genomic DNA was extracted from each isolate using the MiniBEST Bacteria Genomic DNA Extraction Kit (TaKaRa, China) according to the manufacturer's instructions.

2.3. Serotyping. Serotyping by the multiplex PCR assay, as previously reported by Imperi et al. [22], was carried out for each isolate of $S$. agalactiae in this study. Those strains, which could not be classified into any serotype, were designated as nontypeable (NT).

2.4. MLST. Seven housekeeping genes (adhP, pheS, atr, $g \ln A, s d h A, g l c K$, and $t k t)$ were amplified to implement MLST, as described previously [26]. The obtained products were purified and sequenced using sanger sequencing by the Sangon Biotech (Shanghai) Co., Ltd. The allele numbers and STs were obtained using online data (http://pubmlst.org/ sagalactiae/). The phylogenetic tree based on the concatenated sequences of the seven loci was constructed by the MEGA6 software using the UPGMA method. 
The relationships among different STs were estimated using eBURST version 3.1 (http://eburst.mlst.net/v3/). Note that only those shared six identical alleles were defined as a clonal complex (CC) [40, 41]. Additionally, to understand the location of the STs for this study, the eBURST diagram of the $S$. agalactiae population was constructed using all STs found around the world, which are reported in the international database (http://pubmlst.org/sagalactiae/) (see Table S1 in the Supplementary Materials) as of August 2018.

2.5. Antibiotic Susceptibility Tests. All strains were assessed for susceptibility to penicillin (10 units), ampicillin $(10 \mu \mathrm{g})$, erythromycin $(15 \mu \mathrm{g})$, clindamycin $(2 \mu \mathrm{g})$, tetracycline $(30 \mu \mathrm{g})$, levofloxacin $(5 \mu \mathrm{g})$, vancomycin $(30 \mu \mathrm{g})$, linezolid $(30 \mu \mathrm{g})$, and chloramphenicol $(30 \mu \mathrm{g})$ (Oxoid, UK) by disk diffusion method, according to the recommendation of Clinical and Laboratory Standards Institute (CLSI) 2017 guidelines (http://www.clsi.org). The D-zone test was carried out for the strains nonsusceptible to erythromycin but susceptible to clindamycin. Streptococcus pneumoniae ATCC 49619 was used as a quality control strain to ensure the credibility of the results. Multidrug resistance of $S$. agalactiae was defined as acquired nonsusceptibility to at least three classes of antibiotics as previously described $[42,43]$.

2.6. Determination of Macrolides and Lincosamides Resistance Genes. The macrolides resistance genes ermB, ermTR, and $m e f A / E$ were tested by PCR in all erythromycin nonsusceptible isolates, while the lincosamides resistance gene $\operatorname{lin} B$ was detected in each clindamycin-resistant strain. The primers that were used and the conditions in which the reactions were performed were as described previously $[17,44,45]$.

2.7. Detection of Virulence Genes. Five major virulence genes that encode the surface-proteins, including toxins CAMP factor (cfb), $\alpha-(b c a)$ and $\beta$-subunits (bac) of C protein, C5a peptidase $(s c p B)$, and laminin-binding protein $(\operatorname{lmb})$, were analyzed using PCR. The specific experimental procedures used in the present study have been published earlier [29].

\subsection{Characteristic Mass Peak Analysis. MALDI-TOF MS} characteristic mass peak analysis was performed as briefly described below. First, single colonies from the overnightcultured isolates were picked and coated duplicate on a target plate (VITEK ${ }^{\circledR}$ MSDS, bioMérieux, France). $\alpha$-Cyano4-hydroxycinnamic acid (CHCA; bioMérieux, France) $(1 \mu \mathrm{L})$ was added to the tested samples, and the target plate was left to dry. The RUO Mode (VITEK ${ }^{\circledR}$ MS RUO, Shimadzu, France) was then calibrated with Escherichia coli ATCC 8739 strain, and the mass spectrometry data of each strain were collected in a positive linear mode within a mass range from $3,000 \mathrm{Da}$ to $20,000 \mathrm{Da}$ [46]. The data were imported to SARAMIS premium software (bioMérieux, France), and the comparison of the mass peaks was carried out by Lanchpad software (Shimadzu Biotech, USA).
2.9. Statistical and Data Analyses. Fisher's exact test was used to evaluate the differences in antibiotic sensitivity and distribution of resistance genes among different serotypes. The relationships between CCs, serotypes, and related virulence gene profiles were analyzed by a correspondence analysis. All statistical analyses were performed with SPSS software version 22.0. A $P$-value $<0.05$ was considered statistically significant.

2.10. Ethical Statement. This study protocol was in accordance with the Helsinki Declaration of 1964. Data collected in this study did not include the information about patients, which was exempted from the formal medical ethical approval by the ethics committee of the hospital where the research was carried out (ZM2016-280).

\section{Results}

3.1. Serotype Distribution. Four serotypes were detected in $72 \mathrm{~S}$. agalactiae isolates, and serotype III was the most frequently identified serotype, accounting for $52.8 \%$ (38/72). The proportions of serotypes Ia, Ib, and II were $18.0 \%$ (13/ 72), $22.2 \%(16 / 72)$, and $4.2 \%(3 / 72)$, respectively. The other two $S$. agalactiae isolates were NT.

3.2. MLST. Of the 72 S. agalactiae isolates in this study, 71 were classified into 18 unique STs. The allele combination of the remaining strain was adhP (1), pheS (1), atr (4), $g \ln A$ (2), $s d h A(1), g l c K(3)$, and $t k t$ (4). Since the database did not have an assigned ST for this allele combination, the strain could not be classified by MLST. As shown in Figure 1, the most frequently detected ST was ST19 (30.6\%), followed by ST17 (11.1\%), ST485 (8.3\%), ST862 (8.3\%), ST12 (6.9\%), ST651 (6.9\%), and ST27 (5.6\%). The proportions of other STs (ST8, ST23, ST24, ST28, ST86, ST138, ST328, ST824, ST885, ST890, and ST929) were less than 3.0\%, respectively.

Using the eBURST tool, a population snapshot was constructed to show the clusters of all known STs (number of STs = 1311; see Table S1 in the Supplementary Materials) in the entire S. agalactiae MLST database, and the relationship between the STs classified in this study and worldwide prevalent STs (see Figure 2) was elucidated. By eBURST analysis, 18 STs were assigned to 9 CCs (CC8, CC17, CC19, CC23, CC24, CC61, CC103, CC328, and CC485), with CC19 being the most prevalent (45.8\%). The eBURST analysis displayed that CC17, CC61, CC103, CC328, and CC485 were close to CC19. Interestingly, CC19 and CC8 were close to CC1, while CC24 was close to CC23.

3.3. Antibiotic Susceptibility and Resistance Genes. All isolates were sensitive to penicillin, ampicillin, vancomycin, and linezolid. As shown in Table 1, the nonsusceptibility rates for erythromycin, clindamycin, tetracycline, levofloxacin, and chloramphenicol were 69.4\%, 47.2\%, 90.3\%, $37.5 \%$, and $31.9 \%$, respectively. Notably, $66.7 \%$ (48/72) of all S. agalactiae isolates were multidrug resistant (MDR) (see 


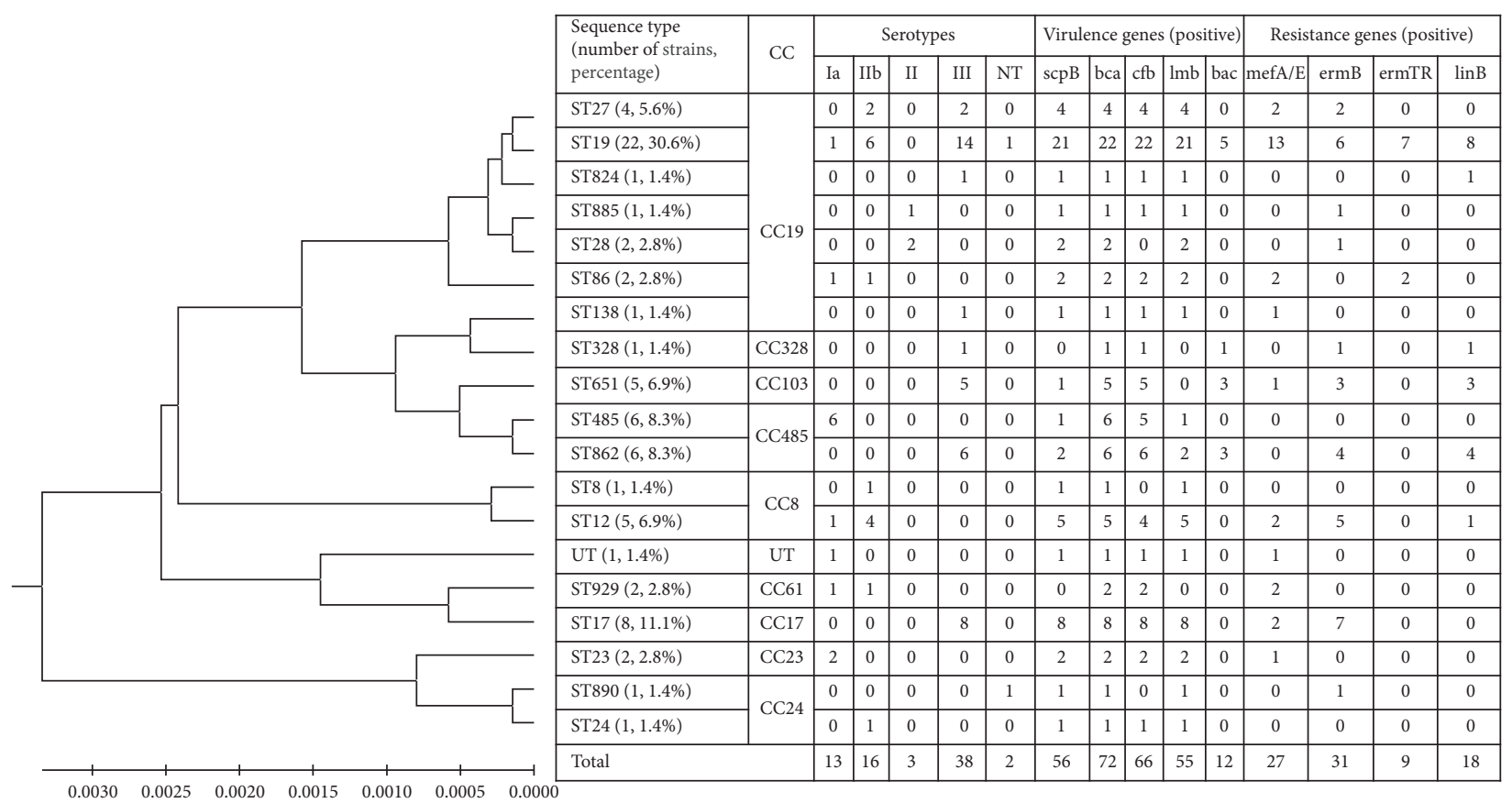

FIgURE 1: UPGMA dendrogram of the 72 S. agalactiae strains was constructed by MEGA6 software. This dendrogram shows the genetic diversity and molecular characteristics of different STs. Nonsusceptibility includes intermediation and resistance, and no strains were found intermediate to clindamycin in this study. ST, sequences type; CC, clonal complex; UT, untypable; NT, nontypeable; values express the number of strains.

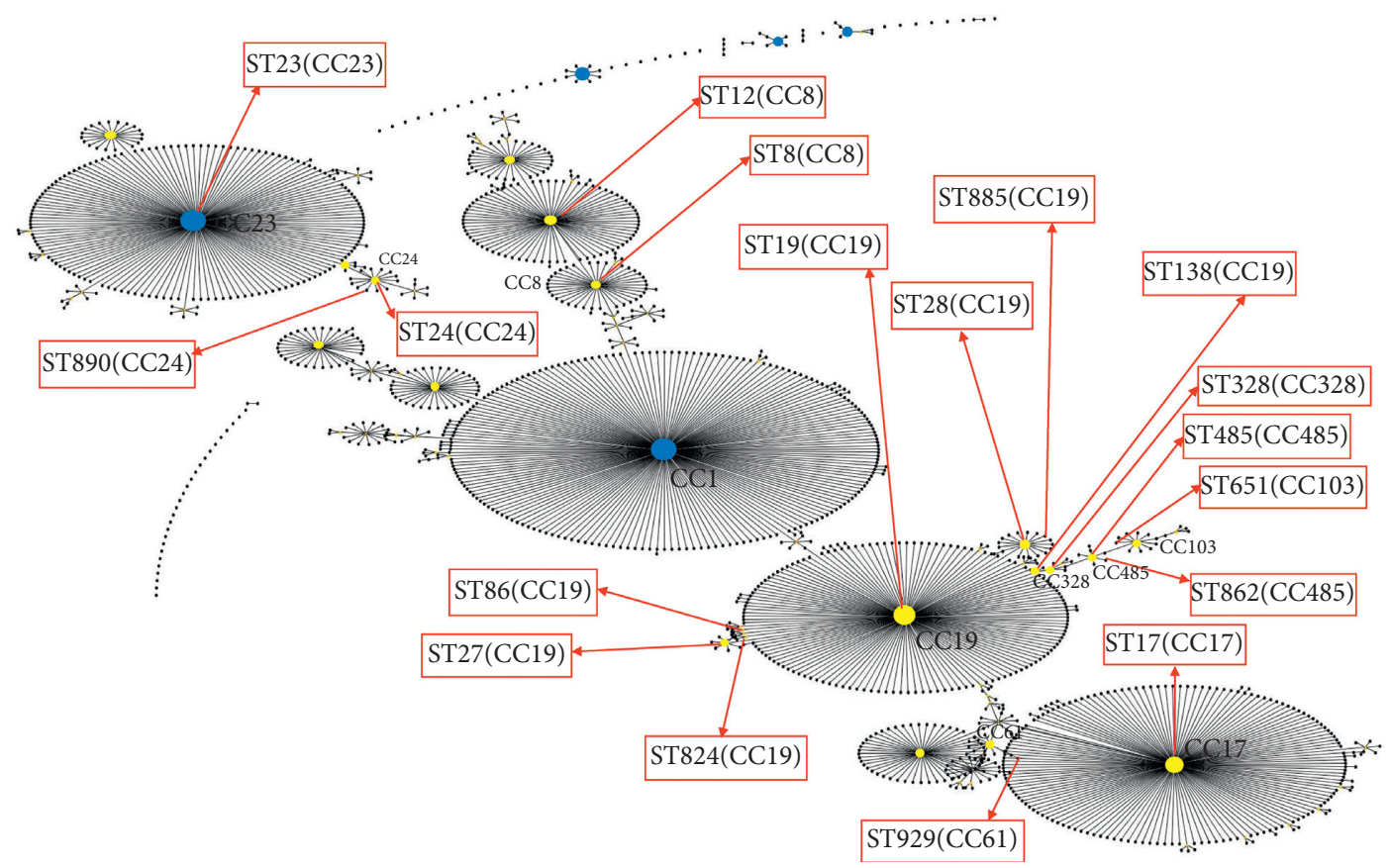

FIgURE 2: The eBURST diagram of S. agalactiae. This diagram is constructed using all STs found around the world as of August 2018. Number of STs $=1311$. ST labels have been removed and the original diagram has been optimized by manual edited. Each dot represents a ST; blue and yellow dots represent founding and subgroup founding types, respectively. The red font represents the clonal complexes found in this study. Red boxes pointed by red arrows indicate the STs detected in this study and display which clonal complexes the STs belongs to.

Table S2 in the Supplementary Materials for antibiotic resistance combination). No strains were found to be intermediate sensitivity to clindamycin and levofloxacin in this study. In other words, the nonsusceptibility rates for clindamycin and levofloxacin were equal to the resistance rates. 19 isolates were nonsusceptible to erythromycin, but 
TAвLE 1: Relationship between antibiotic nonsusceptibility, resistance genes, and serotypes.

\begin{tabular}{|c|c|c|c|c|c|c|c|c|c|c|}
\hline \multirow{2}{*}{ Serotypes } & \multicolumn{6}{|c|}{ Antibiotics $^{\mathrm{a}}$ (nonsusceptibility $^{\mathrm{b}}$ ) } & \multicolumn{4}{|c|}{ Resistance genes (positive) } \\
\hline & ERY & CLI & TET & LEV & CHL & MDR & $\mathrm{ermB}$ & ermTR & $m e f A / E$ & $\operatorname{lin} B$ \\
\hline Total $(n=72)$ & $50(69.4)$ & $34(47.2)$ & $65(90.3)$ & $27(37.5)$ & $23(31.9)$ & $48(66.7)$ & $31(62.0)$ & $9(18.0)$ & $27(54.0)$ & $18(52.9)$ \\
\hline Ia $(n=13)$ & $6(46.2)$ & $3(23.1)$ & $12(92.3)$ & $2(15.4)$ & $3(23.1)$ & $5(38.5)$ & $2(33.3)$ & $2(33.3)$ & $5(83.3)$ & $1(33.3)$ \\
\hline Ib $(n=16)$ & $14(87.5)$ & $6(37.5)$ & $16(100.0)$ & $7(43.8)$ & $7(43.8)$ & $12(75.0)$ & $6(42.9)$ & $6(42.9)$ & $9(64.3)$ & $2(33.3)$ \\
\hline II $(n=3)$ & $2(66.7)$ & $2(66.7)$ & $3(100.0)$ & $0(0.0)$ & $2(66.7)$ & $2(66.7)$ & $2(100.0)$ & $0(0.0)$ & $0(0.0)$ & $0(0.0)$ \\
\hline III $(n=38)$ & $26(65.8)$ & $22(57.9)$ & $32(84.2)$ & $17(44.7)$ & $10(26.3)$ & $27(71.1)$ & $20(76.9)$ & $0(0.0)$ & $12(46.2)$ & $15(68.2)$ \\
\hline $\mathrm{NT}^{\mathrm{c}}(n=2)$ & $2(100.0)$ & $1(50.0)$ & $2(100.0)$ & $1(50.0)$ & $1(50.0)$ & $2(100.0)$ & $1(50.0)$ & $1(50.0)$ & $1(50.0)$ & $0(0.0)$ \\
\hline$P$ value ${ }^{\mathrm{d}}$ & 0.148 & 0.159 & 0.483 & 0.194 & 0.333 & 0.183 & 0.054 & 0.001 & 0.213 & 0.093 \\
\hline
\end{tabular}

Note. Values are $n(\%)$ or as otherwise indicated. ${ }^{a}$ All the strains were sensitive to penicillin, ampicillin, vancomycin, and linezolid. ERY, erythromycin; CLI, clindamycin; TET, tetracycline; LEV, levofloxacin; CHL, chloramphenicol; MDR, multidrug resistant. ${ }^{b}$ Nonsusceptibility includes intermediation and resistance. And no strains were found intermediate to clindamycin and levofloxacin in this study. ${ }^{~}$ Nontypeable. ${ }^{\mathrm{d}} P$-values was calculated with Fisher's exact test.

susceptible to clindamycin, and eight of them were positive for the D-zone test.

The macrolides resistance genes were determined for 50 isolates of erythromycin nonsusceptible S. agalactiae, and at least one macrolides resistance gene was detected in each strain. The major prevalent resistance genes were ermB (62.0\%), followed by mefA/E (54.0\%) and ermTR (18.0\%) (see Table 1). Among the 34 clindamycin-resistant isolates, 18 strains (52.9\%) carried the $\operatorname{lin} B$ gene (see Table 1).

Overall, there were no significant differences in the antibiotic susceptibility, $\operatorname{erm} B, m e f A / E$, and $\operatorname{lin} B$ resistance genes, except in the ermTR gene $(P$-value $=0.001)$, among the different serotype isolates (see Table 1).

3.4. Virulence Genes. As shown in Figure 1, the bca gene was detected in all the strains, and the $c f b$ gene was frequently identified, accounting for $91.7 \%$ (66/72). The prevalence of the $s c p B, l m b$, and $b a c$ virulence genes was $77.8 \%$ (56/72), $70.5 \%(55 / 72)$, and $16.7 \%(12 / 72)$, respectively. Intriguingly, the $\operatorname{lmb}$ gene was detected in almost all the $\operatorname{scp} B$ genepositive strains. Based on the presence or absence of each virulence gene, all 72 strains were clustered into seven virulence gene profiles (see Table 2). The $b c a-c f b-s c p B-l m b$ (61.6\%), bca-cfb (12.5\%), and bca-cfb-bac (9.7\%) patterns were the main virulence gene profiles. Only five strains (6.9\%) contained all five virulence genes.

3.5. Characteristic Mass Peak Analysis. MALDI-TOF MS analysis revealed the differences in the mass peaks among the different STs. Relative to the other STs, all ST17 strains $(n=8)$ were identified at the mass peak of $7620 \mathrm{Da}$ (see Figure S1 in the Supplementary Materials). In other words, the mass peak of $7620 \mathrm{Da}$ was the characteristic mass peak of the ST17 strains.

3.6. Relationships among the Various Molecular Characteristics of S. agalactiae Strains. According to the UPGMA dendrogram, the $S$. agalactiae strains isolated from pregnant women in Guangzhou were divided into two main branches (see Figure 2), namely, CC1 and CC23 (see Figure 1), which were further divided into different subbranches, representing the different STs (see Figure 2). Interestingly, some
STs were closely related to specific serotypes (see Figure 2). For instance, the ST17, ST651, and ST862 strains were attributed to serotype III, while the ST485 and ST885 were strains classified as serotypes Ia and II, respectively.

The correspondence analysis indicated that there was a significant correspondence between the CCs and virulence gene profiles $\left(\chi^{2}=106.52, P\right.$-value $\left.<0.001\right)$ and the serotypes and virulence gene profiles $\left(\chi^{2}=51.61, P\right.$-value $\left.=0.001\right)$ (see Figure 3). For example, the $b c a-c f b-s c p B-\operatorname{lmb}$ profile was distributed in $\mathrm{CC} 17$ and $\mathrm{CC} 23$, the $b c a-c f a-b a c$ profile was distributed in $\mathrm{CC103}$, the $b c a-c f b$ profile was distributed in serotype Ia, and the $b c a-s c p B-\operatorname{lmb}$ profile was distributed in serotype II.

Besides that, all erythromycin nonsusceptible isolates ( $n=50$, including ST27, ST19, ST885, ST28, ST86, ST138, ST651, ST862, ST12, ST929, ST17, ST23, and ST890) carried at least one macrolide resistance gene. Of these strains, $42.0 \%(21 / 50)$ carried the ermB gene, $24.0 \%(12 / 50)$ carried the $m e f A / E$ gene, $16.0 \%(8 / 50)$ carried both the ermB and $m e f A / E$ genes, $4.0 \%(2 / 50)$ carried both the ermB and ermTR genes, and $14.0 \%(7 / 50)$ carried both the mefA/E and ermTR genes (see Table 3). Erythromycin nonsusceptible strains were found in all the mainly prevalent STs (ST12, ST17, ST19, ST27, ST651, and ST862) (see Table 3), except ST485. Some of these mainly prevalent STs were obviously associated with specific macrolide resistance genes. All ST12, ST17, ST651, and ST862 erythromycin nonsusceptible isolates were found to carry the erm $B$ gene (see Table 3 and Figure 1). However, only $31.3 \%$ (5/16) of the ST19 erythromycin nonsusceptibility isolates carried the $\operatorname{erm} B$ gene. The mefA/E gene was significantly related to erythromycin nonsusceptibility in ST19 than in the other mainly prevalent STs (ST12, ST17, ST27, ST651, and ST862) $\left(\chi^{2}=11.99, P\right.$-value $\left.=0.018\right)$, and $81.3 \%(13 / 16)$ of the ST19 erythromycin nonsusceptibility isolates carried the mefA/E gene (see Table 3 and Figure 1). Of the mainly prevalent STs, only some ST19 erythromycin nonsusceptibility isolates were found to carry the ermTR gene (see Table 3 and Figure 1). As mentioned earlier, only $52.9 \%(18 / 34)$ of all clindamycin-resistant isolates were found to carry the $\operatorname{lin} B$ gene. Intriguingly, the linB gene was mainly found in ST19 $(88.9 \%, 8 / 9)$, ST651 (100\%, 3/3), ST862 (100\%, 4/4), ST824 $(100 \%, 1 / 1)$, and ST328 $(100 \%, 1 / 1)$ clindamycin-resistant 
TABLE 2: Virulence gene profiles of 72 S. agalactiae isolates.

\begin{tabular}{|c|c|c|c|}
\hline $\begin{array}{l}\text { Virulence gene } \\
\text { profiles }\end{array}$ & $n(\%)$ & Serotypes (number of isolates) & CC (number of isolates) \\
\hline$b c a$ & $1(1.4 \%)$ & Ia (1) & CC485 (1) \\
\hline$b c a-c f b$ & $9(12.5 \%)$ & Ia (5), Ib (1), and III (3) & CC61 (2), CC103 (1), and CC485 (6) \\
\hline$b c a-c f b-b a c$ & $7(9.7 \%)$ & III $(7)$ & CC19 (1), CC103 (3), CC328 (1), and CC485 (2) \\
\hline$b c a-c f b-s c p B$ & $1(1.4 \%)$ & III (1) & $\mathrm{CC} 103(1)$ \\
\hline$b c a-s c p B-\operatorname{lm} b$ & $5(6.9 \%)$ & Ib (2), II (2), and NT (1) & CC8 (2), CC19 (2), and CC24 (1) \\
\hline$b c a-c f b-s c p B-\operatorname{lm} b$ & $\begin{array}{c}44 \\
(61.1 \%)\end{array}$ & Ia (7), Ib (13), II (1), III (22), and $\mathrm{NT}^{\mathrm{a}}$ & $\begin{array}{c}\mathrm{CC} 8 \text { (4), CC17 (8), CC19 (26), CC23 (2), CC24 (1), CC485 (2), } \\
\text { and } \mathrm{UT}^{\mathrm{b}}(1)\end{array}$ \\
\hline $\begin{array}{l}b c a-c f b-s c p B-\operatorname{lm} b- \\
b a c\end{array}$ & $5(6.9 \%)$ & III (5) & CC19 (4) and CC485 (1) \\
\hline
\end{tabular}

${ }^{\mathrm{a}}$ Nontypeable. ${ }^{\mathrm{b}}$ Untypeable.

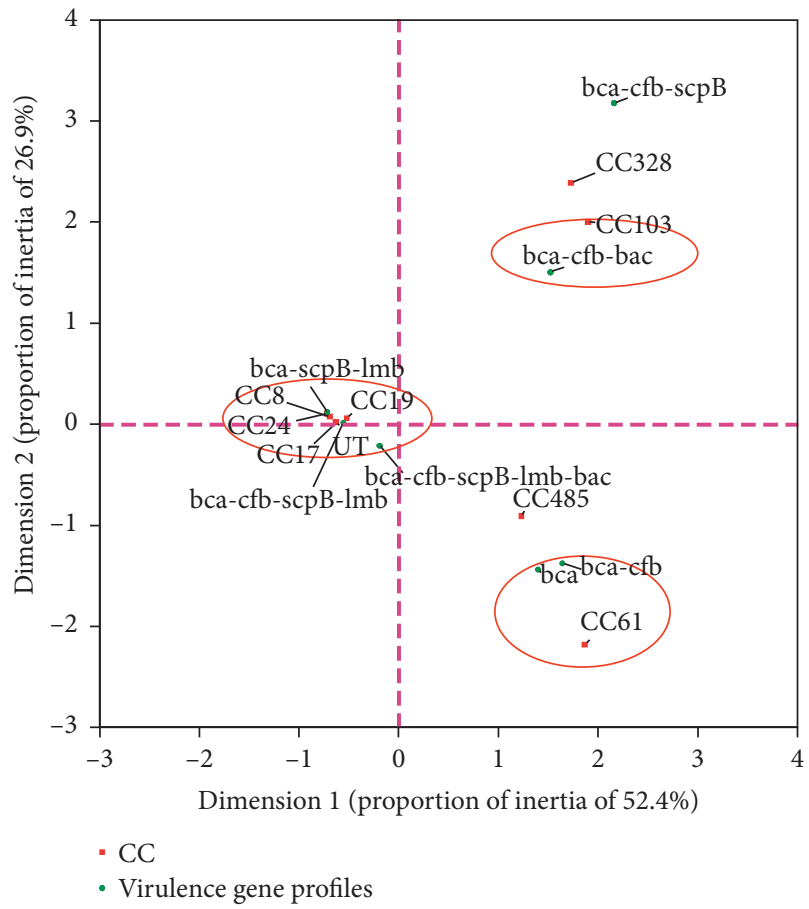

(a)

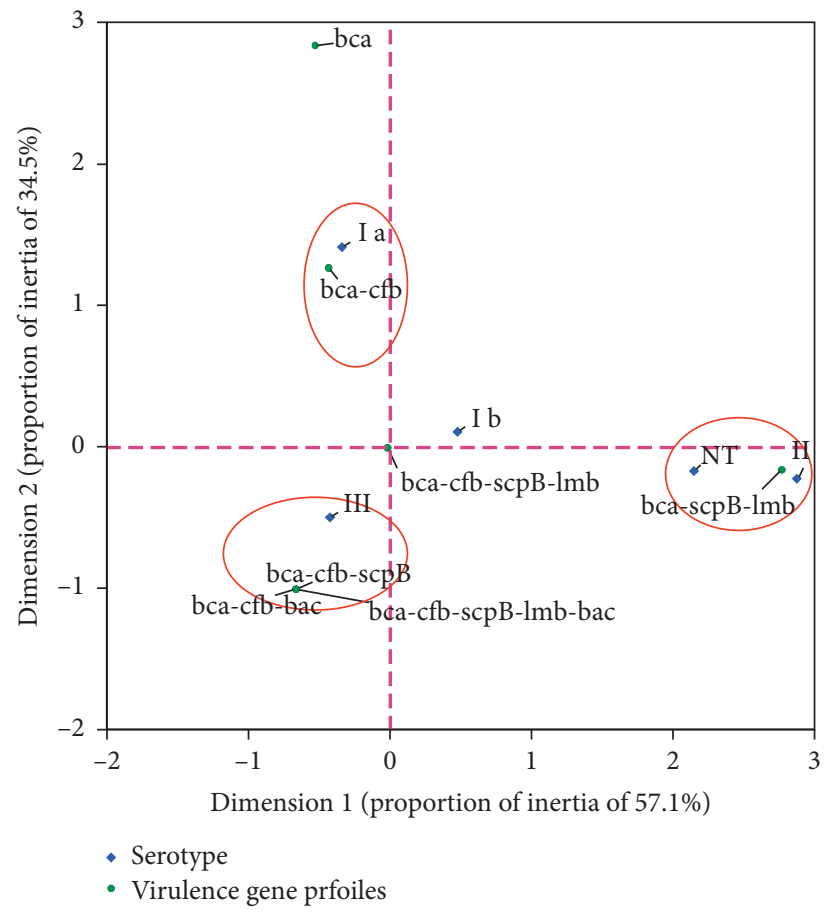

(b)

FIgURE 3: Correspondence analysis for the relationship between CC, serotypes, and virulence gene profiles. (a) CC and virulence gene profiles. (b) Serotypes and virulence gene profiles. CC, clonal complex; UT, untypable; NT, nontypeable.

isolates. However, no $\operatorname{linB}$ gene was found in ST17, ST890, ST885, and ST28 clindamycin-resistant isolates.

\section{Discussion}

Maternal S. agalactiae colonization can lead to postpartum intrauterine infections and invasive neonatal diseases [3-5]. A systematic review and meta-analysis revealed that the carriage rate of S. agalactiae in pregnant women was $10 \%$ (95\% CI 8, 12), with $7 \%$ in Asia and $19 \%$ in non-Asian countries [24]. In this study, the carriage rate of S. agalactiae in pregnant women in Guangzhou was found to be $7.1 \%$. Although the carriage rate was not obviously different from the average rate in Asia, it is necessary to investigate the prevalence of $S$ agalactiae in different regions of China, considering the huge population of China and limited research data of colonized S. agalactiae.

Previous reports have shown differences in the phenotypic and genotypic characteristics of colonized S. agalactiae from different geographical regions [16, 28, 47]. Accordingly, determining the population structure of colonizing isolates (e.g., genetic diversity, virulence factors, and antibiotic resistance) is the key to understand $S$. agalactiae disease in a region [9]. The present study is the first comprehensive investigation of the molecular epidemiological characteristics of colonized isolates in pregnant women in Guangzhou, South China.

Serotyping of $S$. agalactiae is crucial in determining the pathogenicity of the isolates [48]. In this study, four serotypes (Ia, Ib, II, and III) were identified by molecular 
TABLE 3: Association of antibiotic nonsusceptibility and resistance genes with sequence types.

\begin{tabular}{|c|c|c|c|c|c|c|c|c|c|}
\hline \multirow{2}{*}{$\begin{array}{l}\text { ST (number of } \\
\text { strains) }\end{array}$} & \multicolumn{2}{|c|}{$\begin{array}{l}\text { Erythromycin } \\
\text { nonsusceptible }\end{array}$} & \multicolumn{5}{|c|}{ Macrolides resistance genes (positive) } & \multirow{2}{*}{$\begin{array}{l}\text { Clindamycin } \\
\text { resistance }^{\mathrm{a}}\end{array}$} & \multirow{2}{*}{$\begin{array}{c}\operatorname{lin} B \\
\text { (positive) }\end{array}$} \\
\hline & Intermediate & Resistant & ermB & $m e f A / E$ & $\begin{array}{c}e r m B+m e f A / \\
E\end{array}$ & ermB + ermTR & $\begin{array}{c}\text { mefA/E+ } \\
\text { ermTR }\end{array}$ & & \\
\hline ST8 (1) & $0(0.0)$ & $0(0.0)$ & & & & & & $0(0.0)$ & \\
\hline ST24 (1) & $0(0.0)$ & $0(0.0)$ & & & & & & $0(0.0)$ & \\
\hline ST824 (1) & $0(0.0)$ & $0(0.0)$ & & & & & & $1(100.0)$ & $1(100.0)$ \\
\hline ST485 (6) & $0(0.0)$ & $0(0.0)$ & & & & & & $0(0.0)$ & \\
\hline ST27 (4) & $1(25.0)$ & $3(75.0)$ & $2(50.0)$ & $2(50.0)$ & & & & $0(0.0)$ & \\
\hline ST19 (22) & $1(4.5)$ & $15(68.2)$ & $1(6.3)$ & $5(31.2)$ & $3(18.8)$ & $2(12.5)$ & $5(31.3)$ & $9(40.1)$ & $8(88.9)$ \\
\hline ST651 (5) & $0(0.0)$ & $3(60.0)$ & $2(66.7)$ & & $1(33.3)$ & & & $3(60.0)$ & $3(100.0)$ \\
\hline ST862 (6) & $0(0.0)$ & $4(66.7)$ & $\begin{array}{c}4 \\
(100.0)\end{array}$ & & & & & $4(66.7)$ & $4(100.0)$ \\
\hline ST12 (5) & $0(0.0)$ & $5(100.0)$ & $3(60.0)$ & & $2(40.0)$ & & & $5(100.0)$ & $1(20.0)$ \\
\hline ST17 (8) & $0(0.0)$ & 7 (87.5) & $5(71.4)$ & & $2(28.6)$ & & & $7(87.5)$ & \\
\hline ST885 (1) & $0(0.0)$ & $1(100.0)$ & $\begin{array}{c}1 \\
(100.0)\end{array}$ & & & & & $1(100.0)$ & \\
\hline ST28 (2) & $0(0.0)$ & $1(50.0)$ & $\begin{array}{c}1 \\
(100.0)\end{array}$ & & & & & $1(50.0)$ & \\
\hline ST86 (2) & $0(0.0)$ & $2(100.0)$ & & & & & $2(100.0)$ & $0(0.0)$ & \\
\hline ST138 (1) & $0(0.0)$ & $1(100.0)$ & & $\begin{array}{c}1 \\
(100.0)\end{array}$ & & & & $0(0.0)$ & \\
\hline ST328 (1) & $0(0.0)$ & $1(100.0)$ & $\begin{array}{c}1 \\
(100.0)\end{array}$ & & & & & $1(100.0)$ & $1(100.0)$ \\
\hline $\mathrm{UT}^{\mathrm{b}}(1)$ & $0(0.0)$ & $1(100.0)$ & & $\begin{array}{c}1 \\
(100.0)\end{array}$ & & & & $1(100.0)$ & \\
\hline ST929 (2) & $0(0.0)$ & $2(100.0)$ & & $\begin{array}{c}2 \\
(100.0)\end{array}$ & & & & $0(0.0)$ & \\
\hline ST23 (2) & $0(0.0)$ & $1(50.0)$ & & $\begin{array}{c}1 \\
(100.0)\end{array}$ & & & & $0(0.0)$ & \\
\hline ST890 (1) & $0(0.0)$ & $1(100.0)$ & $\begin{array}{c}1 \\
(100.0)\end{array}$ & & & & & $1(100.0)$ & \\
\hline
\end{tabular}

Note. Values are $n(\%)$ or as otherwise indicated. ${ }^{a}$ No strains were found intermediate to clindamycin in this study. ${ }^{\mathrm{b}} \mathrm{Untypeable.}$

serotyping. Overall, the three primary serotypes among the colonized isolates in Guangzhou were III, Ib, and Ia (93.0\%), which were also reported as predominant serotypes in other Chinese cities, such as Dongguan (85.7\%) [11] and Beijing $(66.1 \%)$ [16]. However, the predominant serotypes in many other areas in other reports, including serotypes Ia, II, and Ib in Brazil (65.4\%) [49] and serotypes III, V, and Ia in Toronto (67.0\%) [9] and Shanghai (79.6\%) [21], are different from those in our study. It has been reported that the colonization of serotype III isolates in pregnant women is a high risk factor for neonatal infections $[12,21]$. Notably, the proportion of the most common serotype (serotype III) in our region $(52.8 \%)$ was similar to that reported in adjacent Dongguan (54.9\%) [11], but apparently higher than that reported in Shanghai (East China, accounting for 35.9\%) [21] and Beijing (North China, accounting for 32.1\%) [16]. These data suggest that the prevalence of serotype III isolates in South China may be higher than that in other parts of China. The CPS of $S$. agalactiae is not only a basis for serotyping, but also an important target for vaccine research. In the development of conjugated multivalent vaccines, only five serotypes (Ia, Ib, II, III, and V) were covered [50, 51]. Fortunately, all isolates in this study, except two NT strains, were hypothetically covered by a vaccine combination containing polysaccharide conjugates of the five serotypes.
NT strains have also been reported in previous studies $[2,21,52]$ and may be related to mutations in the CPS locus $[9,53]$.

Molecular epidemiological studies using MLST reveal the characteristics of the distribution of colonized $S$. agalactiae lineages. In this study, 18 distinct STs were identified, and these were then grouped into nine CCs. The colonized isolates from Guangzhou show relatively higher genetic diversity. Nonetheless, the three prevalent CCs, that is, CC19, CC485, and CC17, accounted for $73.6 \%$. CC19 is the most common CC among asymptomatic pregnant women in our region, a finding similar to the global trend [2, 14, 47]. Interestingly, MLST analysis revealed that some bovine dominant subtypes had begun to spread in humans. ST485 was previously thought to be undetectable in humans, but recently, it has dramatically increased in pregnant women in China $(2.5 \%-14.13 \%)[16,33,54]$. In addition, some studies have shown that ST485 is highly pathogenic and may cause threatened abortion, premature rupture of membranes, and early-onset diseases (EOD) $[33,55]$. It is worth noting that ST485 has become one of the leading STs in our region (accounting for 8.6\%). Although all subtypes of CC61 and CC67 were previously reported as bovine strains [47, 53], ST929, which can cause threatened abortion in pregnant women, was first reported as a new subtype of CC67 in 2017 
[33]. Furthermore, $2.8 \%$ of the colonized isolates in our study belong to ST929. The eBURST population snapshot (as of August 2018, number of STs = 1311) (see Figure 2) showed different CCs related to ST485 and ST929, which were previously grouped into CC103 and CC67, respectively [33]. This difference can be explained by the high genetic diversity observed and the dynamic nature of $S$. agalactiae in the MLST database [53].

One of the major objectives of this study was to investigate the gene-based antibiotic resistance of colonized $S$. agalactiae. In the current study, all strains were sensitive to penicillin, which was the first-line antibiotic for the treatment and prevention of $S$. agalactiae infection $[10,11]$. Nevertheless, it is worth noting that our region has a high proportion of MDR strains. Erythromycin and clindamycin have long been considered as effective alternatives in IAP for penicillin-allergic women [9, 17]. However, the resistance rates for erythromycin and clindamycin in our region were $66.7 \%$ and $47.2 \%$, respectively. Recently, such high resistance rates have also been observed in other Chinese cities, such as Beijing [16], Shanghai [29], and Dongguan [11]. Overall, the resistance rates for erythromycin and clindamycin in China were higher than those in other regions $[2,9,18,56]$. Therefore, if erythromycin or clindamycin is used as an alternative antibiotic, antimicrobial susceptibility test should be performed in advance. Erythromycin resistance is usually associated with three macrolide resistance genes: erm $B$, ermTR, and $m e f A / E[17,57]$. Although the erythromycin resistance rates of $S$. agalactiae in different Chinese cities were similar, the resistance mechanisms were not the same. In this study, erythromycin resistance was mediated mainly by the ermB or mef $A / E$ genes, and no isolates were found to carry the ermTR gene alone. The erythromycin-resistant strains carrying resistance genes in our study were different from those in previous reports [57]. The $\operatorname{lin} B$ gene, encoding a lincosamide nucleotidyltransferase, confers moderate sensitivity to erythromycin and resistance to clindamycin $[17,18]$. However, the $\operatorname{lin} B$ gene was detected in two erythromycin-sensitive strains. This may be because the $\operatorname{lin} B$ gene was not expressed, but further research is needed to confirm this inference.

In the current study, five main virulence genes of $S$. agalactiae, which encode the surface proteins that involved in adhesion, invasion, or immune evasion [58], were investigated. Similar to previous reports [27, 28, 49, 59], our report showed that the $c f b, \operatorname{scp} B$, and $\operatorname{lm} b$ genes were distributed in most strains. However, the prevalence of the $b c a$ and bac genes in our study was different from that in previous reports. All strains in our study harbored the $b c a$ gene, indicating that the number of $b c a$-positive strains was significantly higher in our region than that reported in other regions $[29,49,59]$. Our results indicated that the bac gene was identified only in serotype III (Table 2). This result was different from those reported around 2000, which showed that the bac gene was predominantly present in serotypes Ia, Ib, and II $[27,59]$. These results suggested that the epidemiological distribution of $S$. agalactiae virulence genes might vary from period to period and region to region. Dmitriev A et al. used PFGE to show that the bac-positive strains were genetically homogeneous [59]. Instead, we found the that bac gene was distributed in different STs, suggesting that the bac-positive strains were genetically heterogeneous. The result of a previous study that used dotblot hybridization was consistent with our inference [27]. This indicated that when PFGE was used as the major method for determining diversity within a population, the measure of diversity was missed [27]. We found that the $b c a-$ $c f b-s c p B-\operatorname{lmb}$ profile was the most common gene cluster (Table 2), distributed in all serotypes and most CCs. Nevertheless, the correspondence analysis showed that the distribution of virulence gene clusters between the CCs and serotypes was different.

The ST17 strain, belonging to serotype III, was defined as "hypervirulent" S. agalactiae, associated with early onset meningitis and late-onset diseases (LOD) in neonates [39]. With the implementation of extensive screening and IAP strategies, the incidence of EOD has been greatly reduced $[12,39]$. However, the morbidity rates of LOD remain unchanged [12, 39], partly because the ST17 strains are particularly capable of persisting in the vaginal flora [39]. Detection of "hypervirulent" ST17 strains in vaginal samples or in neonates should permit the identification of a neonate population that presents with high risk for $S$. agalactiae infection [60], and in such a population, antimicrobial therapy and strict follow-up are absolutely necessary [60]. In this study, ST17 was one of the most prevalent STs, and all ST17 strains belonged to serotype III. It is necessary to establish a rapid screening method for ST17 strains in pregnant women in this region. MALDI-TOF MS can rapidly distinguish among some bacterial subtypes using characteristic mass spectrum peaks [37]. Previous studies using MALDI-TOF MS (Bruker Daltonics) confirmed the characteristic mass spectrum peak of the ST17 strain at $7625 \mathrm{Da}[2,39]$. However, we found this peak at $7620 \mathrm{Da}$ using VITEK ${ }^{\circledR}$ MS. We speculated that the characteristic mass peaks of different detection systems are slightly different. ST106 was also found to harbor this peak [2], yet we did not identify this ST in this study. We hypothesized that MALDI-TOF MS is suitable for the rapid screening of the ST17 strains in our region.

With the continuous reduction in the cost of WGS and the rapid development of bioinformatic infrastructures [34], it is expected that WGS will be performed in the future to reveal more subtle characteristics of S. agalactiae. This study was limited in its small sample size, single-center design, and retrospective nature. Our next experiment will include an increased sample size. Overall, this study added new information on the antibiotic resistance and molecular characteristics of colonized S. agalactiae in China.

\section{Conclusion}

The data obtained in this study indicated that the main molecular epidemiological characteristics of colonized $S$. agalactiae in Guangzhou are similar to those in other regions. However, some regional characteristics are also shown, especially relatively high prevalence of highly pathogenic ST485 and ST929. Moreover, the high carrying 
rate of the $b c a$ gene and low carrying rate of the $b a c$ gene are also prominent features. This study facilitated the epidemiological comparison of different regions and the prevention of postpartum intrauterine and neonatal infections. Furthermore, due to the high multidrug resistance rate of the colonized strains in our region, anti-infective treatment based on antibiotic resistance monitoring is necessary. Finally, we hypothesized that MALDI-TOF MS is suitable for the rapid screening of the ST17 strains in Guangzhou.

\section{Data Availability}

The data used to support the findings of this study are available from the corresponding author upon request.

\section{Disclosure}

The funding bodies played no role in the research design and implementation or dissemination of findings of the study.

\section{Conflicts of Interest}

The authors declare that there are no conflicts of interest regarding the publication of this paper.

\section{Authors' Contributions}

Zhaomin Cheng, Xianzhang Huang, M.D., Ph.D., and Min He, M.D., Ph.D., conceived and designed the study. Zhaomin Cheng, Nannan Cao, M.D., Xiaohan Yang, and Sheng Qin, M.D., performed the experiments. Peifeng Ke, Qiang Zhou, and Kai Lan conducted statistics and analysis of the data. Zhaomin Cheng and Pinghua Qu, M.D., wrote and revised the manuscript. All authors contributed to the final manuscript. All authors read and approved the final manuscript.

\section{Acknowledgments}

The authors are grateful to the staff at the Microorganism Laboratory of the Guangdong Provincial Maternity and Children's Hospital for their assistance in strains collection. This work was supported by scientific research projects of Traditional Chinese Medicine Bureau of Guangdong Province (Grant no. 20181102) and Guangzhou Science and Technology Project (Grant no. 201704020213).

\section{Supplementary Materials}

Table S1: the MLST profiles of global dataset of S. agalactiae. These data include all S. agalactiae STs found worldwide as of August 2018 (XLS $57 \mathrm{~kb}$ ). Table S2: antibiotic resistance combination of multidrug-resistant colonized $S$. agalactiae (docx 16 kb). Figure S1: characteristic mass spectrum analysis of different ST strains (PDF $271 \mathrm{~kb}$ ). (Supplementary Materials)

\section{References}

[1] N.-Y. Wang, K. A. Patras, H. S. Seo et al., "Group B streptococcal serine-rich repeat proteins promote interaction with fibrinogen and vaginal colonization," Journal of Infectious Diseases, vol. 210, no. 6, pp. 982-991, 2014.
[2] A. Bergal, L. Loucif, D. E. Benouareth, A. A. Bentorki, C. Abat, and J.-M. Rolain, "Molecular epidemiology and distribution of serotypes, genotypes, and antibiotic resistance genes of Streptococcus agalactiae clinical isolates from Guelma, Algeria and Marseille, France," European Journal of Clinical Microbiology \& Infectious Diseases, vol. 34, no. 12, pp. 2339-2348, 2015.

[3] K. A. Patras and V. Nizet, "Group B streptococcal maternal colonization and neonatal disease: molecular mechanisms and preventative approaches," Frontiers in Pediatrics, vol. 6, pp. 1-17, 2018.

[4] B. Rojo-Bezares, J. M. Azcona-gutiérrez, C. Martin, M. S. Jareño, C. Torres, and Y. Sáenz, "Streptococcus agalactiae from pregnant women: antibiotic and heavy-metal resistance mechanisms and molecular typing," Epidemiology and Infection, vol. 144, no. 15, pp. 3205-3214, 2016.

[5] W. Ji, H. Liu, Z. Jin et al., "Disease burden and antimicrobial resistance of invasive group B streptococcus among infants in China: a protocol for a national prospective observational study," BMC Infectious Diseases, vol. 17, no. 1, p. 377, 2017.

[6] J. R. Verani, L. McGee, and S. J. Schrag, "Prevention of perinatal group B streptococcal disease-revised guidelines from CDC, 2010," MMWR Recommendations and Reports, vol. 59, pp. 1-36, 2010.

[7] S. Mukhopadhyay, E. C. Eichenwald, and K. M. Puopolo, "Neonatal early-onset sepsis evaluations among wellappearing infants: projected impact of changes in CDC GBS guidelines," Journal of Perinatology, vol. 33, no. 3, pp. 198205, 2013.

[8] D. Money, V. M. Allen, M. H. Yudin et al., "The prevention of early-onset neonatal group B streptococcal disease," Journal of Obstetrics and Gynaecology Canada, vol. 35, no. 10, pp. 939-948, 2013.

[9] S. Teatero, P. Ferrieri, I. Martin, W. Demczuk, A. McGeer, and N. Fittipaldi, "Serotype distribution, population structure, and antimicrobial resistance of group B Streptococcus strains recovered from colonized pregnant women," Journal of Clinical Microbiology, vol. 55, no. 2, pp. 412-422, 2017.

[10] G. Piccinelli, P. Fernandes, C. Bonfanti, F. Caccuri, A. Caruso, and M. A. De Francesco, "In Vitro activity of solithromycin against erythromycin-resistant Streptococcus agalactiae," Antimicrobial Agents and Chemotherapy, vol. 58, no. 3, pp. 1693-1698, 2014.

[11] W. Ji, L. Zhang, Z. Guo et al., "Colonization prevalence and antibiotic susceptibility of Group B Streptococcus in pregnant women over a 6-year period in Dongguan, China," PLoS One, vol. 12, no. 8, Article ID e0183083, 2017.

[12] J. Liu, R. Xu, H. Zhong et al., "Prevalence of GBS serotype III and identification of a ST 17-like genotype from neonates with invasive diseases in Guangzhou, China," Microbial Pathogenesis, vol. 120, pp. 213-218, 2018.

[13] J. Huang, X.-Z. Lin, Y. Zhu, and C. Chen, "Epidemiology of group $\mathrm{B}$ streptococcal infection in pregnant women and diseased infants in mainland China," Pediatrics \& Neonatology, vol. 60, no. 5, pp. 487-495, 2019.

[14] G. Gherardi, M. Imperi, L. Baldassarri et al., "Molecular epidemiology and distribution of serotypes, surface proteins, and antibiotic resistance among group B streptococci in Italy," Journal of Clinical Microbiology, vol. 45, no. 9, pp. 2909-2916, 2007.

[15] S. M. Garland, E. Cottrill, L. Markowski et al., “Antimicrobial resistance in group B streptococcus: the Australian experience," Journal of Medical Microbiology, vol. 60, no. 2, pp. 230-235, 2011. 
[16] P. Wang, J. J. Tong, X. H. Ma et al., "Serotypes, antibiotic susceptibilities, and multi-locus sequence type profiles of Streptococcus agalactiae isolates circulating in Beijing, China," PLoS One, vol. 10, no. 3, Article ID e0120035, 2015.

[17] S. E. Gygax, J. A. Schuyler, L. E. Kimmel, J. P. Trama, E. Mordechai, and M. E. Adelson, "Erythromycin and clindamycin resistance in group B streptococcal clinical isolates," Antimicrobial Agents and Chemotherapy, vol. 50, no. 5, pp. 1875-1877, 2006.

[18] S. M. Mousavi, M. Nasaj, S. M. Hosseini et al., "Survey of strain distribution and antibiotic resistance pattern of group B streptococci (Streptococcus agalactiae) isolated from clinical specimens," GMS Hygiene and Infection Control, vol. 11, 2016.

[19] H. M. Kang, H. J. Lee, H. Lee et al., "Genotype characterization of group B Streptococcus isolated from infants with invasive diseases in South Korea," The Pediatric Infectious Disease Journal, vol. 36, no. 10, pp. e242-e247, 2017.

[20] K. Yao, K. Poulsen, D. Maione et al., "Capsular gene typing of Streptococcus agalactiae compared to serotyping by latex agglutination," Journal of Clinical Microbiology, vol. 51, no. 2, pp. 503-507, 2013.

[21] Y. Yan, H. Hu, T. Lu et al., "Investigation of serotype distribution and resistance genes profile in group B Streptococcus isolated from pregnant women: a Chinese multicenter cohort study," APMIS, vol. 124, no. 9, pp. 794-799, 2016.

[22] M. Imperi, M. Pataracchia, G. Alfarone, L. Baldassarri, G. Orefici, and R. Creti, "A multiplex PCR assay for the direct identification of the capsular type (Ia to IX) of Streptococcus agalactiae," Journal of Microbiological Methods, vol. 80, no. 2, pp. 212-214, 2010.

[23] K. M. Edmond, C. Kortsalioudaki, S. Scott et al., "Group B streptococcal disease in infants aged younger than 3 months: systematic review and meta-analysis," The Lancet, vol. 379, no. 9815, pp. 547-556, 2012.

[24] J. Huang, S. Li, L. Li, X. Wang, Z. Yao, and X. Ye, “Alarming regional differences in prevalence and antimicrobial susceptibility of group B streptococci in pregnant women: a systematic review and meta-analysis," Journal of Global Antimicrobial Resistance, vol. 7, pp. 169-177, 2016.

[25] S. Li, G. Wen, X. Cao et al., "Molecular characteristics of Streptococcus agalactiae in a mother-baby prospective cohort study: implication for vaccine development and insights into vertical transmission," Vaccine, vol. 36, no. 15, pp. 1941-1948, 2018.

[26] A. Rosenau, K. Martins, S. Amor et al., "Evaluation of the ability of Streptococcus agalactiae strains isolated from genital and neonatal specimens to bind to human fibrinogen and correlation with characteristics of the fbsA and fbsB genes," Infection and Immunity, vol. 75, no. 3, pp. 1310-1317, 2007.

[27] T. C. Smith, S. A. Roehl, P. Pillai, S. Li, C. F. Marrs, and B. Foxman, "Distribution of novel and previously investigated virulence genes in colonizing and invasive isolates of Streptococcus agalactiae," Epidemiology and Infection, vol. 135, no. 6, pp. 1046-1054, 2007.

[28] E. E. Udo, S. S. Boswihi, and N. Al-Sweih, "Genotypes and virulence genes in group B streptococcus isolated in the maternity hospital, Kuwait," Medical Principles and Practice, vol. 22, no. 5, pp. 453-457, 2013.

[29] H. Jiang, M. Chen, T. Li et al., "Molecular characterization of Streptococcus agalactiae causing community- and hospitalacquired infections in Shanghai, China,," Frontiers in Microbiology, vol. 7, 2016.

[30] N. Jones, J. F. Bohnsack, S. Takahashi et al., "Multilocus sequence typing system for group B Streptococcus," Journal of Clinical Microbiology, vol. 41, no. 6, pp. 2530-2536, 2003.
[31] F.-Y. C. Lin, A. Whiting, E. Adderson et al., "Phylogenetic lineages of invasive and colonizing strains of serotype III group B Streptococci from neonates: a multicenter prospective study," Journal of Clinical Microbiology, vol. 44, no. 4, pp. 1257-1261, 2006.

[32] B. Perichon, N. Szili, L. du Merle et al., "Regulation of PI-2b pilus expression in hypervirulent Streptococcus agalactiae ST17 BM110," PLoS One, vol. 12, no. 1, Article ID e0169840, 2017.

[33] L. Li, R. Wang, Y. Huang et al., "High incidence of pathogenic Streptococcus agalactiae ST485 strain in pregnant/puerperal women and isolation of hyper-virulent human CC67 strain," Frontiers in Microbiology, vol. 9, p. 50, 2018.

[34] G. Kapatai, D. Patel, A. Efstratiou et al., "Comparison of molecular serotyping approaches of Streptococcus agalactiae from genomic sequences," BMC Genomics, vol. 18, no. 1, 2017.

[35] B. J. Metcalf, S. Chochua, R. E. Gertz et al., "Short-read whole genome sequencing for determination of antimicrobial resistance mechanisms and capsular serotypes of current invasive Streptococcus agalactiae recovered in the USA," Clinical Microbiology and Infection, vol. 23, no. 8, pp. 574.e7-574.e14, 2017.

[36] N. van der Mee-Marquet, S. M. Diene, L. Barbera et al., "Analysis of the prophages carried by human infecting isolates provides new insight into the evolution of Group B Streptococcus species," Clinical Microbiology and Infection, vol. 24, no. 5, pp. 514-521, 2018.

[37] C.-H. Yang, S. Sauer, A. Freiwald et al., "Classification and identification of bacteria by mass spectrometry and computational analysis," PLoS One, vol. 3, no. 7, Article ID e2843, 2008.

[38] H. C. Lin, J. J. Lu, L. C. Lin et al., "Identification of a proteomic biomarker associated with invasive ST1, serotype VI Group B Streptococcus by MALDI-TOF MS," Journal of Microbiology, Immunology and Infection, vol. 52, no. 1, pp. 81-89, 2019.

[39] M.-F. Lartigue, M. Kostrzewa, M. Salloum et al., "Rapid detection of "highly virulent" group B Streptococcus ST-17 and emerging ST-1 clones by MALDI-TOF mass spectrometry," Journal of Microbiological Methods, vol. 86, no. 2, pp. 262-265, 2011.

[40] C. Beauruelle, A. Pastuszka, P. Horvath et al., "CRISPR: a useful genetic feature to follow vaginal carriage of group B Streptococcus," Frontiers in Microbiology, vol. 8, p. 1981, 2017.

[41] J. Sun, W. Fang, B. Ke et al., "Inapparent Streptococcus agalactiae infection in adult/commercial tilapia," Scientific Reports, vol. 6, no. 1, p. 26319, 2016.

[42] D. Guo, X. Cao, S. Li et al., "Neonatal colonization of group B Streptococcus in China: prevalence, antimicrobial resistance, serotypes, and molecular characterization," American Journal of Infection Control, vol. 46, no. 3, pp. e19-e24, 2018.

[43] A.-P. Magiorakos, A. Srinivasan, R. B. Carey et al., "Multidrug-resistant, extensively drug-resistant and pandrug-resistant bacteria: an international expert proposal for interim standard definitions for acquired resistance," Clinical Microbiology and Infection, vol. 18, no. 3, pp. 268-281, 2012.

[44] L. Zhou, S.-J. Yu, W. Gao, K.-H. Yao, A.-D. Shen, and Y.-H. Yang, "Serotype distribution and antibiotic resistance of 140 pneumococcal isolates from pediatric patients with upper respiratory infections in Beijing, 2010," Vaccine, vol. 29, no. 44, pp. 7704-7710, 2011.

[45] B. Bozdogan, L. Berrezouga, M.-S. Kuo et al., "A new resistance gene, linB, conferring resistance to lincosamides by nucleotidylation in Enterococcus faecium HM1025," Antimicrobial Agents and Chemotherapy, vol. 43, no. 4, pp. 925929, 1999. 
[46] P. E. B. Verwer, W. B. Leeuwen, V. Girard et al., "Discrimination of Aspergillus lentulus from Aspergillus fumigatus by Raman spectroscopy and MALDI-TOF MS," European Journal of Clinical Microbiology \& Infectious Diseases, vol. 33, no. 2, pp. 245-251, 2013.

[47] S. Shabayek and B. Spellerberg, "Group B streptococcal colonization, molecular characteristics, and epidemiology," Frontiers in Microbiology, vol. 9, p. 437, 2018.

[48] M. Mukesi, B. C. Iweriebor, L. C. Obi et al., "Prevalence and capsular type distribution of Streptococcus agalactiae isolated from pregnant women in Namibia and South Africa," BMC Infectious Diseases, vol. 19, no. 1, p. 179, 2019.

[49] V. G. Dutra, V. M. Alves, A. N. Olendzki et al., "Streptococcus agalactiae in Brazil: serotype distribution, virulence determinants and antimicrobial susceptibility," BMC Infectious Diseases, vol. 14, p. 323, 2014.

[50] M. Kobayashi, S. J. Schrag, M. R. Alderson et al., "WHO consultation on group B Streptococcus vaccine development: report from a meeting held on 27-28 April 2016," Vaccine, vol. 37, no. 50, pp. 7307-7314, 2019.

[51] S. M. Lin, Y. Zhi, K. B. Ahn, S. Lim, and H. S. Seo, "Status of group B streptococcal vaccine development," Clinical and Experimental Vaccine Research, vol. 7, no. 1, pp. 76-81, 2018.

[52] M. Morozumi, T. Wajima, M. Takata, S. Iwata, and K. Ubukata, "Molecular characteristics of group B streptococci isolated from adults with invasive infections in Japan," Journal of Clinical Microbiology, vol. 54, no. 11, pp. 26952700, 2016.

[53] G. A. Carvalho-Castro, J. R. Silva, L. V. Paiva et al., "Molecular epidemiology of Streptococcus agalactiae isolated from mastitis in Brazilian dairy herds," Brazilian Journal of Microbiology, vol. 48, no. 3, pp. 551-559, 2017.

[54] B. Lu, D. Wang, H. Zhou et al., "Distribution of pilus islands and alpha-like protein genes of group B Streptococcus colonized in pregnant women in Beijing, China," European Journal of Clinical Microbiology \& Infectious Diseases, vol. 34, no. 6, pp. 1173-1179, 2015.

[55] R. Wang, L. Li, T. Huang et al., "Phylogenetic, comparative genomic and structural analyses of human Streptococcus agalactiae ST485 in China," BMC Genomics, vol. 19, no. 1, p. 716, 2018.

[56] P. A. Nakamura, R. B. B. Schuab, F. P. Neves, C. F. Pereira, G. R. d. Paula, and R. R. Barros, "Antimicrobial resistance profiles and genetic characterisation of macrolide resistant isolates of Streptococcus agalactiae," Memórias do Instituto Oswaldo Cruz, vol. 106, no. 2, pp. 119-122, 2011.

[57] B. Lu, D. Li, Y. Cui, W. Sui, L. Huang, and X. Lu, "Epidemiology of group B streptococcus isolated from pregnant women in Beijing, China," Clinical Microbiology and Infection, vol. 20, no. 6, pp. O370-O373, 2014.

[58] F. P. Lin, R. Lan, V. Sintchenko et al., "Computational bacterial genome-wide analysis of phylogenetic profiles reveals potential virulence genes of Streptococcus agalactiae," PLoS One, vol. 6, no. 4, Article ID e17964, 2011.

[59] A. Dmitriev, Y. Y. Hu, A. D. Shen, A. Suvorov, and Y. H. Yang, "Chromosomal analysis of group B streptococcal clinical strains; bacgene-positive strains are genetically homogenous," FEMS Microbiology Letters, vol. 208, no. 1, pp. 93-98, 2002.

[60] M.-C. Lamy, S. Dramsi, A. Billoët et al., "Rapid detection of the "highly virulent" group B streptococcus ST-17 clone," Microbes and Infection, vol. 8, no. 7, pp. 1714-1722, 2006. 